PROCEEDINGS OF THE

AMERICAN MATHEMATICAL SOCIETY

Volume 140, Number 11, November 2012, Pages 3777-3783

S 0002-9939(2012)11228-6

Article electronically published on March 14, 2012

\title{
REPEATED AND FINAL COMMUTATORS IN GROUP ACTIONS
}

\author{
I. M. ISAACS AND ULRICH MEIERFRANKENFELD
}

(Communicated by Jonathan I. Hall)

\begin{abstract}
Let $G$ be a finite group and suppose that $A$ acts via automorphisms on $G$. The repeated commutators are the subgroups $[G, A, A, \ldots, A]$, where there is some positive number of commutations by $A$, and the final commutator is the smallest of these repeated commutators. We show that if $[G, A]$ is nilpotent, then the final commutator is normal in $G$. Also, in general, if $K$ is an arbitrary repeated commutator and $P$ is the permutation group induced by the action of $A$ on the left cosets of $K$ in $G$, we relate the structure of $P$ to the structure of $[G, A]$.
\end{abstract}

\section{INTRODUCTION}

Let $G$ be a group and suppose that some group $A$ acts on $G$ via automorphisms. We define the repeated commutators of $G$ by $A$ to be the subgroups of the form $[G, A, A, \ldots, A]$, where the number of commutations by $A$ is positive but otherwise arbitrary. (These repeated commutators were called the "displacement groups of $G$ under $A$ " by I. J. Mohamed in [4.) We will always assume that $G$ is finite, and hence there is a smallest repeated commutator, and we call this the final commutator of $G$ by $A$.

It is well known that if $X$ and $Y$ are arbitrary subgroups of some group $\Gamma$, then $X$ and $Y$ normalize the commutator $[X, Y]$. Applying this where $\Gamma=G A$, the semidirect product, we see that all of the repeated commutators of $G$ by $A$ are $A$ invariant, and they are subnormal in $G$. They are not necessarily normal, however, except, of course, that $[G, A]$ is always normal.

The situation where the final commutator is the trivial subgroup has been well studied. For example, Philip Hall [1] proved that in this case, both $[G, A]$ and $A / \mathbf{C}_{A}(G)$ are nilpotent. Hall also showed that the nilpotence class of $A / \mathbf{C}_{A}(G)$ is at most $r(r-1) / 2$, where $r$ is the number of commutations by $A$ required to reach the (trivial) final commutator. (Hall's bound on the nilpotence class of $A / \mathbf{C}_{A}(G)$ was subsequently improved by T. C. Hurley [2.)

Our goal in this paper is to study the repeated commutators of $G$ by $A$ without assuming that the final commutator is trivial. It is convenient, therefore, to paraphrase Hall's results so as to avoid this assumption. The group $A / \mathbf{C}_{G}(A)$ is, of course, isomorphic to the group of permutations induced by the action of $A$ on the elements of $G$, so if the final commutator is trivial, Hall's theorem asserts that this permutation group is nilpotent. In general, and whether or not a repeated commutator $K$ is trivial, we know that $K$ is $A$-invariant, and so $A$ permutes the

Received by the editors July 21, 2010 and, in revised form, May 3, 2011.

2010 Mathematics Subject Classification. Primary 20D45, 20D35, 20 D30.

Key words and phrases. Automorphism group, commutator, nilpotent group, SHP-class.

(C)2012 American Mathematical Society 
set of left cosets of $K$ in $G$, and we consider the group $P(G, A, K)$ of permutations induced by $A$ on this set of cosets. We mention that since taking inverses yields a natural bijection between the set of left cosets of $K$ in $G$ and the set of right cosets of $K$ in $G$, the actions of $A$ on these two sets are permutation isomorphic. Thus although we have chosen to work with left cosets, it follows that we could have defined $P(G, A, K)$ as the permutation group induced by $A$ on the set of right cosets of $K$ in $G$, and there would be no essential difference.

In the case where $K=1$, Hall's theorem asserts that both $[G, A]$ and $P(G, A, 1)$ are nilpotent. We show that these two conclusions are related in the following way. Without assuming that the final commutator is trivial, we prove that if $[G, A]$ is nilpotent and $K$ is an arbitrary repeated commutator of $G$ by $A$, then the permutation group $P(G, A, K)$ is nilpotent.

In proving Hall's theorem, where we have $K=1$, we see that once it is established that $[G, A]$ is nilpotent (which seems to be the easier of Hall's two conclusions) one could appeal to our result to deduce that $A / \mathbf{C}_{A}(G) \cong P(G, A, 1)$ is nilpotent. This approach is not entirely satisfactory, however, because it does not yield an upper bound on the nilpotence class of $A / \mathbf{C}_{A}(G)$ as was established by Hall.

In fact, we can weaken the hypothesis that $[G, A]$ is nilpotent and still deduce that $P(G, A, K)$ is nilpotent. To state our result, we recall the notation $G^{\infty}$ to denote the final term of the lower central series of a finite group $G$. Thus $G$ is nilpotent if and only if $G^{\infty}=1$.

Theorem A. Let $A$ act via automorphisms on a finite group $G$ and let $K$ be a repeated commutator of $G$ by $A$. Assume that $[G, A]$ is nilpotent, or more generally that $[G, A]^{\infty} \subseteq K$. Then the permutation group $P(G, A, K)$ induced by $A$ on the set of left cosets of $K$ in $G$ is nilpotent.

Actually, nilpotence is largely irrelevant both in the hypothesis and in the conclusion of Theorem A; it can be replaced by membership in what we shall call an SHP-class of finite groups. By this, we mean a class $\mathcal{X}$ of groups that is closed under taking subgroups, homomorphic images and products of normal subgroups. (The latter condition means that if $U, V \triangleleft G$ and both $U$ and $V$ lie in $\mathcal{X}$, then $U V \in \mathcal{X}$.) Nilpotent groups, solvable groups and $p$-solvable groups are examples of SHP-classes. Also, more generally than solvable or $p$-solvable groups, the class of groups with the property that every simple section is isomorphic to a member of some specifed set of simple groups is an SHP-class. (By a section of a group $G$, we mean a group of the form $U / V$, where $V \triangleleft U \subseteq G$.) Other SHP-classes include solvable groups with Fitting height at most $n$ and $p$-solvable groups with $p$-length at most $n$, where $n \geq 0$ is some given integer. (It is clear that these classes are closed under subgroups and homomorphic images, and we leave it to the reader to check that they are also closed under products of normal subgroups.) We shall sometimes refer to a member of a class $\mathcal{X}$ as an $\mathcal{X}$-group, and finally, we note that neither the class of abelian groups nor the class of supersolvable groups is an SHP-class.

If $\mathcal{X}$ is an SHP-class and $U, V \triangleleft G$ are such that $G / U$ and $G / V$ are $\mathcal{X}$-groups, then $G /(U \cap V)$ is isomorphic to a subgroup of the $\mathcal{X}$-group $(G / U) \times(G / V)$, and thus $G /(U \cap V)$ is an $\mathcal{X}$-group. It follows that given a finite group $G$, there exists a unique smallest normal subgroup $N$ such that $G / N \in \mathcal{X}$, and we write $N=G^{\mathcal{X}}$. Note that if $\mathcal{X}$ is the class of nilpotent groups, then $G^{\mathcal{X}}=G^{\infty}$. The following includes Theorem A. 
Theorem B. Let $A$ act via automorphisms on a finite group $G$. Suppose $K$ is a repeated commutator of $G$ by $A$, and assume that $[G, A]^{\mathcal{X}} \subseteq K$ for some SHP-class $\mathcal{X}$. Then the permutation group $P(G, A, K)$ is an $\mathcal{X}$-group.

If $K$ is an arbitrary $A$-invariant subgroup of $G$, where $A$ acts on $G$ via automorphisms, then of course, the permutation group $P(G, A, K)$ is isomorphic to $A / N$, where $N$ is the kernel of the action of $A$ on the set of left cosets of $K$ in $G$. To prove Theorem B, therefore, it suffices to show that $A^{\mathcal{X}} \subseteq N$, and so we need to determine the kernel $N$. The following easy observation suffices for this purpose.

Lemma C. Let $A$ act on $G$ via automorphisms and let $K \subseteq G$ be $A$-invariant. Then the kernel of the action of $A$ on the set of left cosets of $\bar{K}$ in $G$ is the unique largest normal subgroup $N$ of $A$ such that $[G, N] \subseteq K$.

Proof. An element $a \in A$ lies in the kernel of the action on the set of left cosets of $K$ in $G$ if and only if $(g K)^{a}=g K$, for all $g \in G$, or equivalently, if and only if $g^{-1} g^{a} \in K$ for all $g \in G$. Since $g^{-1} g^{a}=[g, a]$, the result folllows.

In the situation of Theorem $\mathrm{B}$, it follows that to prove that $P(G, A, K)$ is an $\mathcal{X}$-group, we must show that $\left[G, A^{\mathcal{X}}\right] \subseteq K$.

We consider next the question of when the final commutator of $G$ by $A$ is normal in $G$. If $M$ is an arbitrary $A$-invariant normal subgroup of $G$, there is a natural action of $A$ on $\bar{G}=G / M$, and it is easy to see that $[\bar{G}, A]=\overline{[G, A]}$, and more generally, if $K$ is any repeated commutator of $G$ by $A$, then $\bar{K}$ is a repeated commutator of $\bar{G}$ by $A$.

Now suppose that the final commutator $K$ of $G$ by $A$ is normal in $G$ and write $\bar{G}=G / K$. Then the repeated commutator $\bar{K}$ of $\bar{G}$ by $A$ is the trivial subgroup, and we are in Hall's situation, so we know that $[\bar{G}, A]=\overline{[G, A]}$ is nilpotent, and thus $[G, A]^{\infty} \subseteq K$. This part of Hall's theorem, therefore, can be paraphrased, as follows.

Theorem D (Hall). Let $A$ act by automorphisms on a finite group $G$, and let $K$ be the final commutator of $G$ by $A$. If $K \triangleleft G$, then $[G, A]^{\infty} \subseteq K$.

When the final commutator $K$ is normal in $G$ as in Theorem D, the kernel of the action of $A$ on the left cosets of $K$ in $G$ is exactly $\mathbf{C}_{A}(\bar{G})$. The permutation group $P(G, A, K)$ is therefore isomorphic to $A / \mathbf{C}_{A}(\bar{G})$, and we know that this group is nilpotent. (This follows by Hall's original result or by Theorem $\mathrm{D}$ together with our Theorem A.)

The following is a converse to Theorem D.

Theorem E. Let $A$ act on a finite group $G$, and let $K$ be the final commutator of $G$ by $A$. If $[G, A]^{\infty} \subseteq K$, then $K \triangleleft G$.

We mention that if in Theorem $\mathrm{E}$, we strengthen the condition that $[G, A]^{\infty} \subseteq K$, and we assume instead that the derived subgroup $[G, A]^{\prime} \subseteq K$, then not only is the final commutator $K$ normal in $G$, but in fact, all repeated commutators of $G$ by $A$ are normal in $G$. This follows by Theorem 1 of [4, which asserts that if $U \triangleleft G$, where $U$ is a repeated commutator, then $[U, A] \triangleleft G$ if and only if $[G, A, U] \subseteq[U, A]$.

Although the final commutator of $G$ by $A$ is not always normal in $G$, the following result shows that the normalizer of the final commutator is always relatively large.

Theorem F. Let $A$ act via automorphisms on a finite group $G$, with final commutator $K$. Then the generalized Fitting subgroup $\mathbf{F}^{*}(G)$ normalizes $K$. 
Recall that $\mathbf{F}^{*}(G)=\mathbf{F}(G) \mathbf{E}(G)$, where the layer $\mathbf{E}(G)$ is the product of all components of $G$. It is a standard result (see 6.9.2 of [3]) that components normalize all subnormal subgroups, and thus $\mathbf{E}(G)$ normalizes $K$. The force of Theorem $\mathrm{F}$, therefore, is that the Fitting subgroup $\mathbf{F}(G)$ always normalizes the final commutator.

\section{SHP-CLASSES AND SUBNORMAL SUBGROUPS}

All groups in the following are assumed to be finite. We begin with an easy result.

2.1. Lemma. Supppose that $\mathcal{X}$ is an SHP-class.

(a) Let $H \subseteq G$. Then $H^{\mathcal{X}} \subseteq G^{\mathcal{X}}$.

(b) Let $N \triangleleft G$ and write $\bar{G}=G / N$. Then $\bar{G}^{\mathcal{X}}=\bar{G}^{\mathcal{X}}$.

Proof. For (a), we have

$$
H /\left(H \cap G^{\mathcal{X}}\right) \cong H G^{\mathcal{X}} / G^{\mathcal{X}} \subseteq G / G^{\mathcal{X}},
$$

and since $G / G^{\mathcal{X}}$ is an $\mathcal{X}$-group, it follows that $H /\left(H \cap G^{\mathcal{X}}\right)$ is also an $\mathcal{X}$-group. Then $H^{\mathcal{X}} \subseteq H \cap G^{\mathcal{X}} \subseteq G^{\mathcal{X}}$, as wanted.

For (b), observe first that

$$
\bar{G} / \overline{G^{\mathcal{X}}}=\frac{G / N}{N G^{\mathcal{X}} / N} \cong G / N G^{\mathcal{X}},
$$

and this is a homomorphic image of the $\mathcal{X}$-group $G / G^{\mathcal{X}}$. It follows that $\bar{G} / \overline{G^{\mathcal{X}}}$ is an $\mathcal{X}$-group, and thus $\bar{G}^{\mathcal{X}} \subseteq{\overline{G^{\mathcal{X}}}}^{\text {. }}$

To obtain the reverse containment, write $\bar{G}^{\mathcal{X}}=\bar{M}$, with $M \supseteq N$. Then

$$
G / M \cong \bar{G} / \bar{M}=\bar{G} / \bar{G}^{\mathcal{X}}
$$

which is an $\mathcal{X}$-group. Then $G^{\mathcal{X}} \subseteq M$, so $\overline{G^{\mathcal{X}}} \subseteq \bar{M}=\bar{G}^{\mathcal{X}}$.

2.2. Lemma. Let $\mathcal{X}$ be an SHP-class, and suppose $G=U N$, where $N \triangleleft G$ and $U \triangleleft \triangleleft G$. If $N$ is an $\mathcal{X}$-group, then $G^{\mathcal{X}}=U^{\mathcal{X}}$.

Proof. Suppose first that $U \triangleleft G$, and note that $U^{\mathcal{X}} \subseteq G^{\mathcal{X}}$ by Lemma 2.1(a). For the reverse containment, observe that $U^{\mathcal{X}} \triangleleft G$, and write $\bar{G}=G / U^{\mathcal{X}}$. Now $\bar{G}=\bar{U} \bar{N}$ is a product of two normal $\mathcal{X}$-subgroups, so $\bar{G}$ is an $\mathcal{X}$-group. Then $G^{\mathcal{X}} \subseteq U^{\mathcal{X}}$, as required. This shows that $G^{\mathcal{X}}=U^{\mathcal{X}}$ if $U \triangleleft G$.

For the general case, we proceed by induction on $|G: U|$. We can assume that $U<G$, and thus there exists $M \triangleleft G$ with $U \subseteq M<G$. Then $G=M N$, so $G^{\mathcal{X}}=M^{\mathcal{X}}$ by the result of the first paragraph with $M$ in place of $U$. Also, $M=U(N \cap M)$ by Dedekind's lemma, and $N \cap M$ is a normal $\mathcal{X}$-subgroup of $M$. By the inductive hypothesis, we have $M^{\mathcal{X}}=U^{\mathcal{X}}$, and the result follows.

2.3. Lemma. Let $\mathcal{X}$ be an SHP-class, and suppose $G=U V$, where $U \triangleleft \triangleleft G$ and $V \triangleleft \triangleleft G$. Then $G^{\mathcal{X}}=U^{\mathcal{X}} V^{\mathcal{X}}$.

Proof. First, suppose that $V \triangleleft G$. Then $V^{\mathcal{X}} \triangleleft G$, and we write $\bar{G}=G / V^{\mathcal{X}}$. Then $\bar{V}$ is an $\mathcal{X}$-group, so by Lemma 2.1(b) and Lemma 2.2, we have

$$
\overline{G^{\mathcal{X}}}=\bar{G}^{\mathcal{X}}=\bar{U}^{\mathcal{X}}=\overline{U^{\mathcal{X}}}
$$


and thus $G^{\mathcal{X}} \subseteq U^{\mathcal{X}} V^{\mathcal{X}}$. The reverse containment is clear, so we are done in this case.

For the general case, use induction on $|G: V|$. We can assume that $V<G$, and we choose $M \triangleleft G$ with $V \subseteq M<G$. Then $G=U M$, and so $G^{\mathcal{X}}=U^{\mathcal{X}} M^{\mathcal{X}}$ because $M$ is normal. Also, $M=(M \cap U) V$, so the inductive hypothesis yields $M^{\mathcal{X}}=(M \cap U)^{\mathcal{X}} V^{\mathcal{X}} \subseteq U^{\mathcal{X}} V^{\mathcal{X}}$. Thus $G^{\mathcal{X}}=U^{\mathcal{X}} M^{\mathcal{X}} \subseteq U^{\mathcal{X}} V^{\mathcal{X}}$. The reverse containment is clear.

One might ask what happens if we weaken the assumption that $G=U V$ in Lemma 2.3 and assume only that $G=\langle U, V\rangle$. We have been unable to decide if it is possible to conclude that $G^{\mathcal{X}}=\left\langle U^{\mathcal{X}}, V^{\mathcal{X}}\right\rangle$ in this situation.

\section{The MAIN THEOREMS}

We begin with a routine observation.

3.1. Lemma. Let $A$ act on $U$ via automorphisms and write $V=[U, A]$. Working in the semidirect product $U A$, we have $V A \triangleleft U A$.

Proof. Since $A$ certainly normalizes $V A$, it suffices to show that $U$ normalizes $V A$, or equivalently, that $[V A, U] \subseteq V A$. As $V \triangleleft U A$, we can work in $\overline{U A}=U A / V$, and we have

$$
\overline{[V A, U]}=[\overline{V A}, \bar{U}]=[\bar{A}, \bar{U}]=\overline{[A, U]}=\bar{V},
$$

where the second equality holds because $\bar{V}=1$. Then $[V A, U] \subseteq V \subseteq V A$, as required.

Now suppose that $A$ acts on $G$ via automorphisms, and let $\Gamma=G A$ be the semidirect product. Recall that each repeated commutator $K$ of $G$ by $A$ is $A$ invariant, and thus $K A$ is a subgroup of $\Gamma$. Note also that by Dedekind's lemma, $K A \cap G=K(A \cap G)=K$. We have the following.

3.2. Lemma. Let $K$ be a repeated commutator of $G$ by $A$, and let $L=K A$. Then $L \triangleleft \triangleleft \Gamma$, and if $K$ is the final commutator, then $L$ is the subnormal closure of $A$ in $\Gamma$.

Proof. Since $K$ is a repeated commutator, we can write $G=K_{0} \supseteq K_{1} \supseteq K_{2} \supseteq$ $\cdots \supseteq K_{r}=K$, where $K_{i}=\left[K_{i-1}, A\right]$ for $0<i \leq r$. To show that $L=K A$ is subnormal in $\Gamma=G A$, it suffices to show that $K_{i} A \triangleleft K_{i-1} A$ for $0<i \leq r$. This follows from Lemma 3.1 by setting $U=K_{i-1}$ and $V=[U, A]=K_{i}$.

Now assume that $K$ is the final commutator of $G$ by $A$, so $[K, A]=K$. If $L$ is not the subnormal closure of $A$ in $\Gamma$, there exists $M \triangleleft L$ such that $A \subseteq M<$ $L=K A$, and we see that $K \nsubseteq M$. Since $K=[K, A] \subseteq[K, M] \subseteq M$, we have a contradiction.

3.3. Corollary. Let $K$ be a repeated commutator of $G$ by $A$ and let $L=K A$. If $\mathcal{X}$ is an SHP-class, then $[G, A]^{\mathcal{X}} L^{\mathcal{X}} \triangleleft \Gamma$.

Proof. Lemma 3.1 yields $[G, A] L=[G, A] K A=[G, A] A \triangleleft \Gamma$, and thus $([G, A] L)^{\mathcal{X}} \triangleleft$ $\Gamma$. Since $L \triangleleft \triangleleft \Gamma$ by Lemma 3.2, we have $[G, A]^{\mathcal{X}} L^{\mathcal{X}}=([G, A] L)^{\mathcal{X}}$, and the result follows. 
We can now prove Theorem B, which, we recall, includes Theorem A.

Proof of Theorem B. By hypothesis, $[G, A]^{\mathcal{X}} \subseteq K$, where $K$ is a repeated commutator of $G$ by $A$ and $\mathcal{X}$ is an SHP-class. Our goal is to show that the permutation group $P(G, A, K)$ is an $\mathcal{X}$-group, and by Lemma $\mathrm{C}$, we know that it suffices to show that $\left[G, A^{\mathcal{X}}\right] \subseteq K$. Writing $L=K A$ and $N=[G, A]^{\mathcal{X}} L^{\mathcal{X}} \subseteq K L=L$, we have $N \triangleleft \Gamma$ by Corollary 3.3. Also, $A^{\mathcal{X}} \subseteq L^{\mathcal{X}} \subseteq N$, and so $\left[G, A^{\mathcal{X}}\right] \subseteq G \cap N \subseteq G \cap L=K$, as wanted.

Proof of Theorem E. Here, $K$ is the final commutator, and as before, we write $L=K A$. By Lemma 3.2, there does not exist a subgroup $M \triangleleft L$ with $A \subseteq M<L$, and since maximal subgroups of $L$ that contain $L^{\infty}$ are normal in $L$, it follows that $L=L^{\infty} A$.

Write $N=K A^{\infty}$, so $L=N A$, and observe that $N$ is normalized by $A$, so $N \triangleleft L$. Also $L / N=N A / N=A /(A \cap N)$ is nilpotent since $A^{\infty} \subseteq A \cap N$, and thus $L^{\infty} \subseteq N$, and we have $A^{\infty} \subseteq L^{\infty} \subseteq N=K A^{\infty}$. Dedekind's lemma now yields $L^{\infty}=\left(L^{\infty} \cap K\right) A^{\infty}$. Then $K \subseteq L=L^{\infty} A=\left(L^{\infty} \cap K\right) A$, so

$$
K \subseteq\left(L^{\infty} \cap K\right) A \cap G=L^{\infty} \cap K \subseteq L^{\infty} \cap G \subseteq L \cap G=K,
$$

and we have $K=L^{\infty} \cap G$, and in particular, $K \subseteq L^{\infty}$.

By hypothesis, $[G, A]^{\infty} \subseteq K \subseteq L^{\infty}$, so by Corollary 3.3 , we have $L^{\infty}=$ $[G, A]^{\infty} L^{\infty} \triangleleft \Gamma$, and thus $K=L^{\infty} \cap G \triangleleft \Gamma$ and the proof is complete.

Proof of Theorem $F$. Here, $K$ is the final commutator of $G$ by $A$, and we must show that $\mathbf{F}^{*}(G)=\mathbf{F}(G) \mathbf{E}(G)$ normalizes $K$. As we remarked in Section 1, the layer $\mathbf{E}(G)$ normalizes $K$ since $K \triangleleft \triangleleft G$, so it suffices to prove that $\mathbf{F}(G)$ normalizes $K$.

Write $F=\mathbf{F}(G)$, and note that $F$ is $A$-invariant and normal in $G$, and thus $A$ acts on the group $F K$. The repeated commutators of $F K$ by $A$ are contained in the corresponding commutators of $G$ by $A$, and so some repeated commutator of $F K$ by $A$ is contained in $K$. However $[K, A]=K$, and thus $K$ is the final commutator of $F K$ by $A$. Also since $F$ is nilpotent, $(F K)^{\infty}=K^{\infty} \subseteq K$ by Lemma 2.2 , and it follows that $[F K, A]^{\infty} \subseteq K$. By Theorem E applied with $F K$ in place of $G$, it follows that $K \triangleleft F K$, so $F$ normalizes $K$, as required.

\section{An example}

Suppose we try to strengthen Theorem B by relaxing somewhat the condition that $\mathcal{X}$ is an SHP-class. For example, consider the class $\mathcal{X}$ of groups whose composition factors all lie in some given set of simple groups. Then $\mathcal{X}$ is not necessarily closed under taking subgroups, but it is closed under taking subnormal subgroups, as well as under homomorphic images and products of normal subgroups. Also for all finite groups $G$, the $\mathcal{X}$-residual $G^{\mathcal{X}}$ is well defined. Nevertheless, as the following example shows, the analog of Theorem B can fail in this case.

Let $S$ be an arbitrary nonabelian simple group, and let $\mathcal{X}$ be the class of groups with the property that every composition factor is isomorphic to $S$. Let $G$ be the wreath product of $S$ by a cyclic group of order 2 . Then $G$ has a normal subgroup $N$ of index 2, and $N=S_{1} \times S_{2}$, where each factor is isomorphic to $S$, and the $S_{i}$ are conjugate in $G$. Let $A$ be an arbitrary nonidentity proper subgroup of $S_{1}$, and let $A$ act on $G$ by conjugation. Since $A \subseteq N$, we see that $[G, A] \subseteq N$, and $[G, A]>1$ since $A$ is not central in $G$. Then $[G, A]$ is a nonidentity normal subgroup of $G$ contained 
in $N$, and it follows that $[G, A]=N$. Now $A \subseteq S_{1} \triangleleft N$, so $[G, A, A]=[N, A] \subseteq S_{1}$, and thus $[G, A, A]=S_{1}$ since $S_{1}$ is minimal normal in $N$ and $A$ is not central in $N$. It follows by the simplicity of $S_{1}$ that $S_{1}$ is the final commutator of $G$ by $A$.

Now $[G, A]^{\mathcal{X}}=N^{\mathcal{X}}=1 \subseteq S_{1}$, but the permutation group $P\left(G, A, S_{1}\right)$ does not lie in $\mathcal{X}$. To see this, observe first that by Lemma $\mathrm{C}$, this permutation group is a nontrivial homomorphic image of $A$ since $[G, A] \nsubseteq S_{1}$. Thus $1<\left|P\left(G, A, S_{1}\right)\right| \leq$ $|A|<|S|$, so $P\left(G, A, S_{1}\right)$ has composition factors different from $S$, and thus it does not lie in $\mathcal{X}$.

\section{REFERENCES}

1. P. Hall, "Some sufficient conditions for a group to be nilpotent". Illinois J. Math. 2 (1958), 787-801. MR0105441 (21:4183)

2. T. C. Hurley, "On the class of the stability group of a series of subgroups". J. London Math. Soc. (2) 41 (1990), 33-41. MR.1063540 (91m:20053)

3. H. Kurzweil and B. Stellmacher, The theory of finite groups. Springer-Verlag, New York (2004). MR2014408 (2004h:20001)

4. I. J. Mohamed, "On series of subgroups related to groups of automorphisms". Proc. London Math. Soc. (3) 13 (1963), 711-723. MR0155903(27:5836)

Department of Mathematics, University of Wisconsin, 480 Lincoln Drive, Madison, WISCONSIN 53706

E-mail address: isaacs@math.wisc.edu

Department of Mathematics, Michigan State University, East Lansing, Michigan 48824

E-mail address: meier@math.msu.edu 